\title{
Superposition Coding to Support Multiple Streams, Priorities, and Channel Capacities in the Context of GMSK
}

\author{
Thomas A. Courtade, Jiadong Wang, and Richard D. Wesel \\ Department of Electrical Engineering \\ University of California, Los Angeles \\ Los Angeles, California 90095 \\ Email: \{tacourta,wjd, wesel\}@ ee.ucla.edu
}

\begin{abstract}
It is well known in information theory that superposition coding is an optimal technique for providing a single transmission that both provides a high data rate when channel conditions are good and still provides some information when the channel conditions are poor (perhaps due to jamming). Typical superposition coding schemes for the additive white Gaussian noise channel combine signals after the modulator. For modulation schemes such as GMSK, this standard form of (additive) superposition is not acceptable because it does not maintain a constant envelope. This paper introduces a new approach in which newly designed nonlinear codes allow superposition to take place before the GMSK modulation. These codes are demonstrated to operate very close to the boundary of the achievable rate region for the broadcast binary symmetric channel which would result from GMSK transmission with hard decoding. The results also suggest that similarly optimal performance might be obtainable in the soft decoding regime.
\end{abstract}

\section{INTRODUCTION}

In tactical networks, the signal-to-noise ratio (SNR) of the communication channel is frequently unknown (due to a dynamic environment, adversarial jamming, etc.). However, even if the channel quality is poor, it is desirable to maintain some measure of connectivity so that users have access to critical information. Moreover, in cases where the channel quality is relatively good, it is also desirable for the receiver to have access to additional, non-critical information. Thus, the challenge is to design a transmission scheme in which a receiver can decode a stream of critical data in all anticipated channel conditions, and can also recover an additional stream of non-critical data when the channel quality is relatively good.

One way to approach this problem is to consider the two-receiver ${ }^{1}$ degraded broadcast channel (DBC) in which a transmitter broadcasts a common transmission to two different receivers. In the case of the DBC, it is assumed that the signal received at the second receiver is a degraded (i.e., noisier) version of the signal received at the first receiver. Typically, two independent data streams at rates $R_{1}$ and $R_{2}$ respectively are encoded together and broadcast to the receivers. The

This research was supported by Rockwell Collins through contract \#4502769987.

${ }^{1}$ We restrict our discussion to the two-receiver degraded broadcast channel in this paper, however the results can be extended to more users. first receiver is able to decode both data streams (for a total information rate $R_{1}+R_{2}$ ), while the second (degraded) receiver is only able to decode the second data stream at rate $R_{2}$. By encoding critical information into the second data stream at rate $R_{2}$ and non-critical information into the first data stream at rate $R_{1}$, we can ensure that a receiver has access to the critical information when the channel quality is poor, and that receivers can recover additional, non-critical information at rate $R_{1}$ when the channel quality is sufficiently good.

For many DBCs (in particular the AWGN-DBC), an optimal technique for achieving any point in the capacity region is called superposition coding. In superposition coding, the two data streams are independently encoded and modulated, and the modulated signals are scaled and added together. Receivers decode the streams through successive decoding. For some modulation schemes, such as $m$-ary QAM, this approach is perfectly acceptable. However, many tactical waveforms employ constant-envelope modulation (e.g., GMSK), and this additive superposition of modulated signals is unacceptable because it destroys the constant-envelope characteristics of the modulation scheme.

In this paper, we present a coding scheme (based on nonlinear codes) which circumvents this problem by performing the superposition prior to the modulation. Thus, our encoding scheme supports the independent encoding of two distinct data streams (which are recoverable at different channel qualities), while the modulation and spectral characteristics of the transmitted signal remain unchanged.

This paper is organized as follows. In Section II-A, we introduce the fundamentals of nonlinear turbo-codes. In Section II-B, we formally define the DBC and give capacity results for the special case of the binary-symmetric DBC. A systematic technique for designing nonlinear turbo-codes is described in Section III. Section IV presents the architecture for our system and also provides simulation results, demonstrating that our scheme is nearly optimal. Section V delivers the conclusions and discusses directions for future work. 

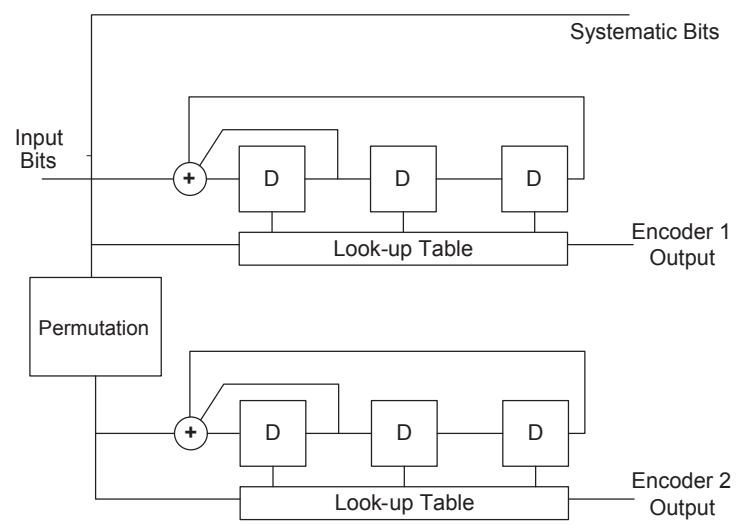

Fig. 1. Block diagram representation of a nonlinear turbo-encoder.

\section{BACKGROUND}

In this section, we provide the background material required to describe our superposition coding approach. In the first subsection, we briefly review the concepts behind nonlinear turbo-codes, and in the second subsection we formally define the degraded broadcast channel.

\section{A. Fundamentals of Nonlinear Turbo-Codes}

Nonlinear turbo-codes, similar to their linear counterparts, consist of constituent encoders connected via interleavers. The key difference between nonlinear and linear turbo-codes is the following: In a linear turbo code, the output bits from a consituent encoder are linear combinations of the encoder state and the input bits. In a nonlinear turbo-code, the output bits from a constituent encoder correspond to the output of a lookup-table addressed by the encoder state and the input bits. See Fig. 1 for a block diagram of a nonlinear turbo-code.

The critical advantage afforded by nonlinear turbo-codes is their capability of producing codewords with ones densities other than $50 \%$. As we will demonstrate in the remainder of this paper, the ability to design a nonlinear turbo-code with a desired ones density allows one to independently encode different data streams, XOR the codewords together, send the modulated sum over a channel, and then successively decode to recover one or both of the streams depending on channel quality. As we noted in Section I, this strategy preserves the constellation of the original modulation scheme, which is a requirement for constant-envelope modulations such as GMSK.

A systematic method for designing nonlinear turbo-codes is given in Section III.

\section{B. Introduction to the $D B C$}

In this section, we define the Degraded Broadcast Channel (DBC) and introduce the Broadcast Binary Symmetric Channel (BBSC), which will be of particular interest to us in Section IV.

Definition 1 (e.g., [1]): A broadcast channel consists of an input alphabet $\mathcal{X}$ and two output alphabets $\mathcal{Y}_{1}$ and $\mathcal{Y}_{2}$ and a

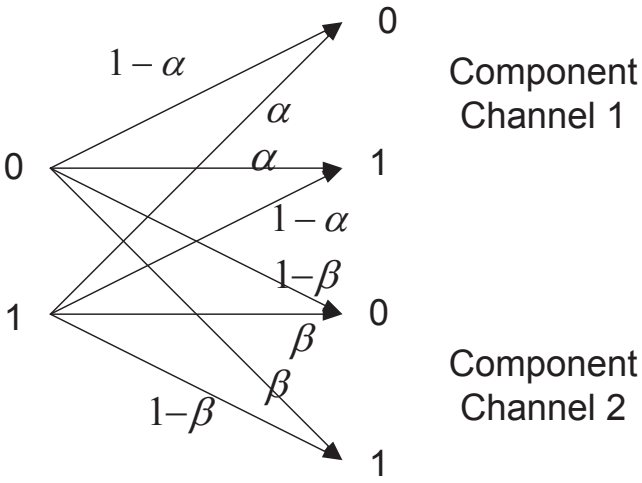

Fig. 2. Channel model for two-user broadcast binary-symmetric channel.

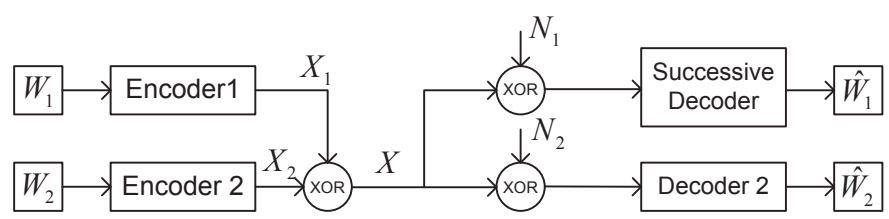

Fig. 3. Encoder scheme for two-user broadcast binary-symmetric channel.

probability transition function $p\left(y_{1}, y_{2} \mid x\right)$. The broadcast channel is said to be stochastically degraded (or just degraded) if there exists a distribution $p^{\prime}\left(y_{2} \mid y_{1}\right)$ such that

$$
p\left(y_{2} \mid x\right)=\sum_{y_{1}} p\left(y_{1} \mid x\right) p^{\prime}\left(y_{2} \mid y_{1}\right) .
$$

In this paper, we will restrict our attention to memoryless broadcast channels, i.e. $p\left(y_{1}^{n}, y_{2}^{n} \mid x^{n}\right)=\prod_{i=1}^{n} p\left(y_{1 i}, y_{2 i}, x_{i}\right)$.

Of particular interest to us is the BBSC, which is sufficient to model any binary modulation scheme in which the demodulator provides hard decisions to the decoder. The two-user BBSC consists of two binary symmetric component channels, one with transition probability $\alpha$ and the other with transition probability $\beta$, as shown in Fig. 2. Without loss of generality, we assume $\alpha<\beta$. A simple and optimal encoding scheme is an independent-encoding approach in which binary codewords from independent codebooks are added together using the XOR operation [2]-[7]. We refer to this scheme as superposition coding, and the encoder structure is shown in Fig. 3.

The capacity region of a degraded broadcast channel was established by Cover [1], Bergmans [8] and Gallager [9]. Cover [10] introduced an independent-encoding scheme for two-user broadcast channels. This scheme is known to achieve the boundary of the capacity region for the broadcast binarysymmetric channel (BBSC) and is investigated in [2] [3] [4] [5]. The class of channels for which independent encoding is optimal was recently extended in [6], [7].

The capacity region for a BBSC is given by

$$
\begin{aligned}
& R_{1} \leq h(\alpha * \rho)-h(\alpha) \\
& R_{2} \leq 1-h(\beta * \rho),
\end{aligned}
$$


where $h(\cdot)$ is the binary entropy function, $\rho$ is the ones density of $X_{1}, X_{2}$ has $50 \%$ ones density and the operation $*$ is defined by

$$
a * b=a(1-b)+b(1-a), \quad 0<a, b<1 .
$$

In order to use a superposition coding scheme with $R_{2}>0$, the codes of the two users cannot both have ones densities of $50 \%$. This precludes the exclusive use of linear codes. Using the techniques described in Section III, we design a family of nonlinear turbo codes that can provide a controlled ones density. Superposition of one of our nonlinear codes with a linear turbo code produces an overall transmission with the potential to approach an optimal point the capacity region of the BBSC.

\section{A Design Scheme for Nonlinear Turbo Codes}

A nonlinear turbo code is defined by look-up tables that map state and input bits to output bits in the trellises of the constituent codes. By controlling the number of ones in the look-up table, any desired ones density can be closely approximated. In general, a brute-force search to find the look-up table yielding the largest effective free distance is impractical because the complexity grows exponentially with the number of states.

In this section, we provide a systematic method for designing nonlinear turbo codes with a complexity that does not grow exponentially in the number of states. Therefore, our approach can be used to design codes employing trellises with many states. We would like to remark that although our technique is described in the context of binary turbo codes that use identical constituent encoders, it immediately extends to more general cases.

\section{A. State sub-tables, branch distance, and merge distance}

Throughout this section, we consider a trellis corresponding to a constituent code of the turbo code. We assume that the trellis has $\ell$ states and each trellis transition corresponds to $k$ input bits and $n$ output bits. Using this notation, we define a state sub-table.

Definition 2: A state sub-table $M(s)$ corresponding to state $s$ is a $2^{k}$-by- $n$ binary matrix describing the mapping of input-bits to output-bits when the encoder is in state $s$ as follows. When the encoder is in state $s$ and the inputbits are $b_{1}, \ldots, b_{k}$, the encoder outputs the $n$ bits in the row corresponding to the $k$-tuple $b_{1}, \ldots, b_{k}$. Without loss of generality, we assume that the rows of $M(s)$ are indexed by the binary $k$-tuples in lexicographical order.

We also define the minimum branch-distance and the minimum merge-distance as follows:

Definition 3: The branch-distance of state $s$ is the minimum distance between the rows of $M(s)$, the branch-distance of a trellis is the minimum of the state branch-distances. The merge-distance of a state and the merge-distance of a trellis are defined analogously as the minimum distances between the $n$-bit outputs of trellis transitions that merge into a state.

As discussed above, designing the constituent codes for a nonlinear turbo code includes defining a look-up table that maps state and input bits to output bits in a trellis. A lookup table that defines a constituent code with a large effective free distance is preferable to one that defines a constituent code with a small effective free distance. A good heuristic for determining whether a look-up table will produce a code with a large effective free distance is to analyze the distances at the branches and merges of the trellis. Our approach is based on the following key observation:

Observation 1: If $\Pi_{1}$ and $\Pi_{2}$ are $2^{k} \times 2^{k}$ and $n \times n$ permutation matrices respectively, then a state sub-table $M\left(s^{\prime}\right)=$ $\Pi_{1} M(s) \Pi_{2}$ has the same branch distance properties as $M(s)$ in the sense that the set of distances between outputs from state $s$ is the same as the set of distances between outputs from state $s^{\prime}$.

\section{B. Description of Design Scheme}

With this observation in mind, we now describe our design approach:

1) If we require a constituent code with ones density $u_{1}$, choose $\nu$ to be the nearest integer to $u_{1} \cdot n \cdot 2^{k}$.

2) Select parameters $d_{b}$ and $d_{m}$, where $d_{b}$ is the desired branch-distance of the trellis and $d_{m}$ is the desired merge-distance of the trellis.

3) Create a state sub-table $M(1)$ with exactly $\nu$ ones. This ensures that the average ones density of the output bits from state 1 is $\frac{\nu}{n \cdot 2^{k}}$ which is approximately $u_{1}$. The state sub-table is designed by carefully placing the ones so that the branch-distance is greater than $d_{b}$. If this is not possible, then return to step 2 and select a smaller $d_{b}$. This step is the main source of complexity. If $n$ and $k$ are sufficiently small, a good $M(1)$ can be found via a brute-force search.

4) For each other state $s \in\{2, \ldots, \ell\}$, choose random permutation matrices $\Pi_{1}$ and $\Pi_{2}$ and set $M(s)=$ $\Pi_{1} M(1) \Pi_{2}$. By Observation 1, this ensures that the trellis has branch-distance greater than $d_{b}$.

5) Check the resulting merge-distance of the trellis. If it is less than $d_{m}$, return to step 4 . If a maximum number of iterations is reached, return to step 2 and select a smaller $d_{m}$.

6) Check the effective free distance of the obtained code. Steps 3-5 are generally repeated several times to produce several candidate codes. Usually, we select the code with the largest effective free distance.

With this scheme, we can construct nonlinear turbo codes without an exhaustive computer search over all lookup tables with the desired ones density. Since we only manually design a sub-table for one state, the complexity does not grow exponentially in the number of states. Through a series of many experiments, we have observed that this procedure is effective for designing nonlinear turbo codes that approach capacity in channels demanding nonuniform ones densities. In the remainder of this paper, we use this technique to design nonlinear turbo codes for BBSCs.

Further details on nonlinear turbo code design, along with applications to a variety of channels, can be found online [11]. 


\section{System ARchitecture And Results}

In this section we apply the idea of superposition coding for the BBSC to develop a coding architecture that supports the encoding of two independent data streams at different priorities. We design the encoder so that both streams can be decoded when channel quality is good, and the high-priority data stream can be decoded even when the channel quality is poor. Furthermore, this encoding scheme preserves the original modulated constellation and is therefore suitable for constantenvelope modulations, such as GMSK. Simulation results are given to demonstrate the near optimality of our codes.

Our approach can be extended to encode more than two independent data streams at different priorities, but this more general case is not discussed in order to simplify the presentation.

\section{A. System Architecture}

The architecture for our coding system is identical, in principle, to that for the BBSC. In particular, we consider two independent data streams which we refer to as the High-Priority Data (HPD) and the Low-Priority Data (LPD). We design our encoders so that the HPD can be recovered whenever $E_{s} / N_{0}$ is greater than some threshold $\eta_{1}$ and the LPD can be recovered whenever $E_{s} / N_{0}$ is greater than $\eta_{2}$ (we assume $\eta_{1}<\eta_{2}$ ). If the rate of the LPD is $R_{1}$ and the rate of the HPD is $R_{2}$, then the critical design parameter is $\rho$, the ones density of the codewords for the LPD encoder. This density determines the $E_{s} / N_{0}$ thresholds $\eta_{1}$ and $\eta_{2}$ via the tradeoff described by (1). As an example, the evolution of the thresholds $\eta_{1}$ and $\eta_{2}$ vs. the ones density $\rho$ is shown in Fig. 4 for $\left(R_{1}, R_{2}\right)=(0.1,0.408)$.

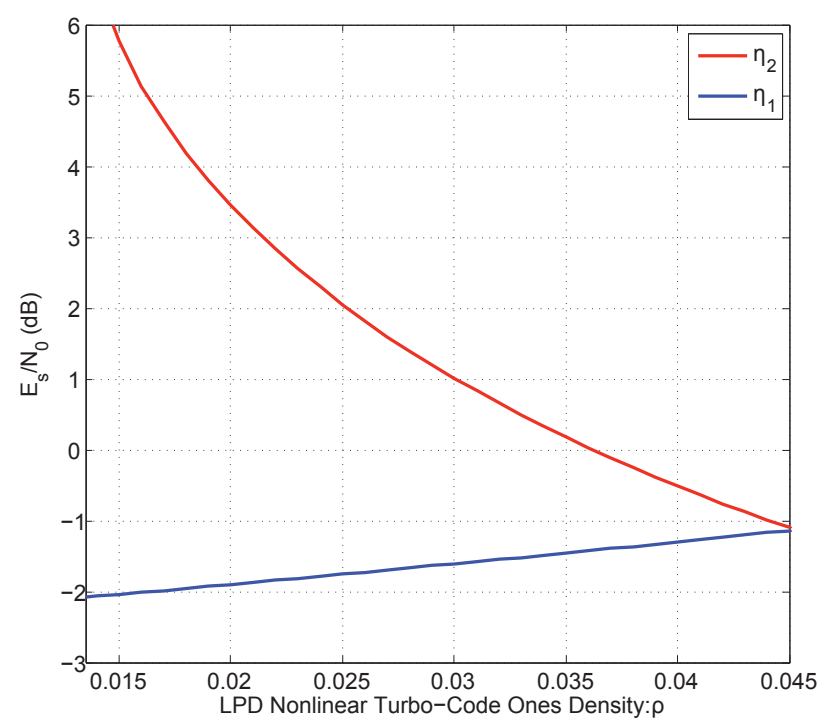

Fig. 4. The relationship between $\eta_{1}$ and $\eta_{2}$ versus $\rho$ for choice of rates $R_{1}=0.1$ and $R_{2}=0.408$.

The encoder/modulator implemented in this work consists of one linear and one nonlinear turbo-encoder joined via the XOR operation. The XOR of the outputs from the encoders are

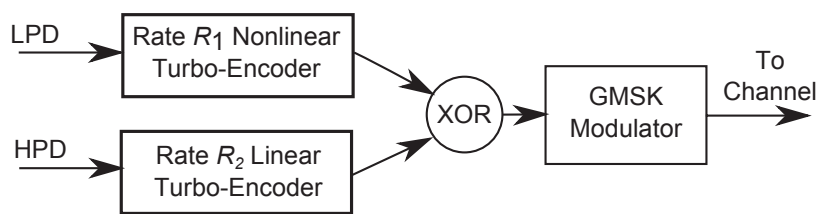

Fig. 5. Superposition Encoder/Modulator Architecture.

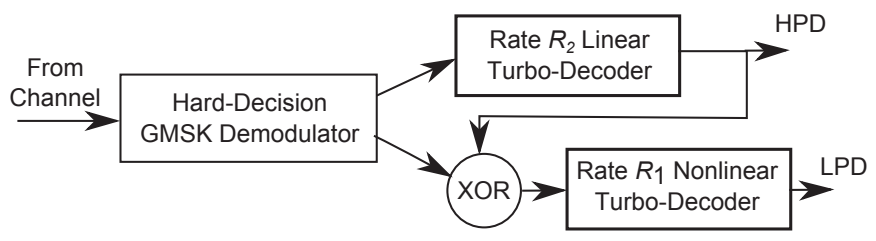

Fig. 6. Superposition Demodulator/Decoder Architecture.

fed into a GMSK modulator with modulation index $h=1 / 2$, $B T=0.3$, and differential precoding. The encoder/modulator architecture is shown in Fig. 5.

The demodulator/decoder consists of an optimal harddecision GMSK demodulator followed by a linear turbodecoder which, in turn, is successively followed by the nonlinear turbo-decoder. The demodulator/decoder architecture is shown in Fig. 6.

\section{B. Simulation Results}

In this section, we present simulation results for two distinct choices of code rates. Each case requires a nonlinear turbocode with rate $R_{1}$ and ones density $\rho$. These codes were designed using the techniques described in Section III. All simulations were performed using an AWGN channel.

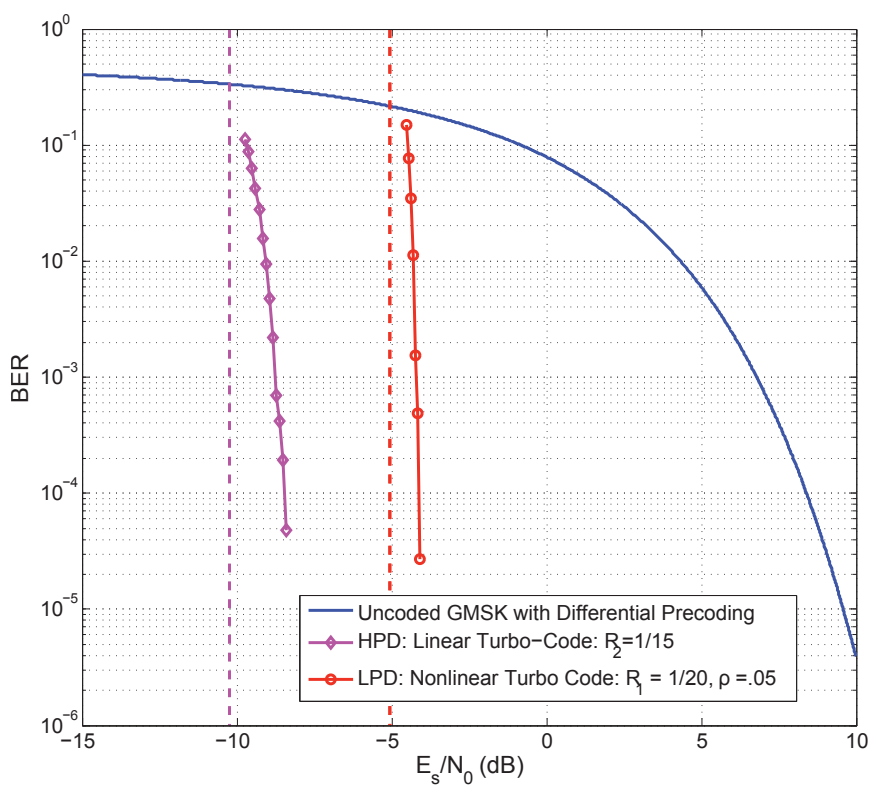

Fig. 7. BER Curves for the HPD and LPD codes at rates $R_{2}=1 / 15$ and $R_{1}=1 / 20$ respectively versus $E_{s} / N_{0}$. Theoretical capacities $\left(\eta_{1}\right.$ and $\left.\eta_{2}\right)$ for the respective codes are given by vertical dashed lines. 
Case 1: $R_{1}=1 / 20, R_{2}=1 / 15, \rho=0.05:$ In this case, we used a rate- $1 / 20$ nonlinear turbo-code with $\rho=0.05$ and blocklength 80,000 for the LPD, and a rate- $1 / 15$ linear turbo code with blocklength 7,500 for the HPD. In both cases, we also designed extended spread interleavers for each turbo code [12]. In Fig. 7 we show the BER curves resulting from simulating our codes using GMSK over an AWGN channel. For a BER of $10^{-4}$, the HPD and LPD codes are approximately $1.8 \mathrm{~dB}$ and $1.0 \mathrm{~dB}$ away from capacity respectively. From this figure we see three distinct operating regions in which none, the HPD only, or the HPD and LPD data streams can be reliably decoded.

Case 2: $R_{1}=0.3, R_{2}=0.2, \rho=0.125:$ In this case, we used a rate- 0.3 nonlinear turbo-code with $\rho=0.125$ and blocklength 10,000 for the LPD, and a rate- 0.2 linear turbo code with blocklength 2,500 for the HPD. In both cases, we also designed extended spread interleavers for each turbo code. In Fig. 8 we show the BER curves resulting from simulating our codes using GMSK over an AWGN channel. For a BER of $10^{-4}$, the HPD and LPD codes are approximately $2.1 \mathrm{~dB}$ and $1.5 \mathrm{~dB}$ away from capacity respectively. The slight loss in performance relative to Case 1 can be explained by the significantly shorter blocklengths and higher code rates which are used.

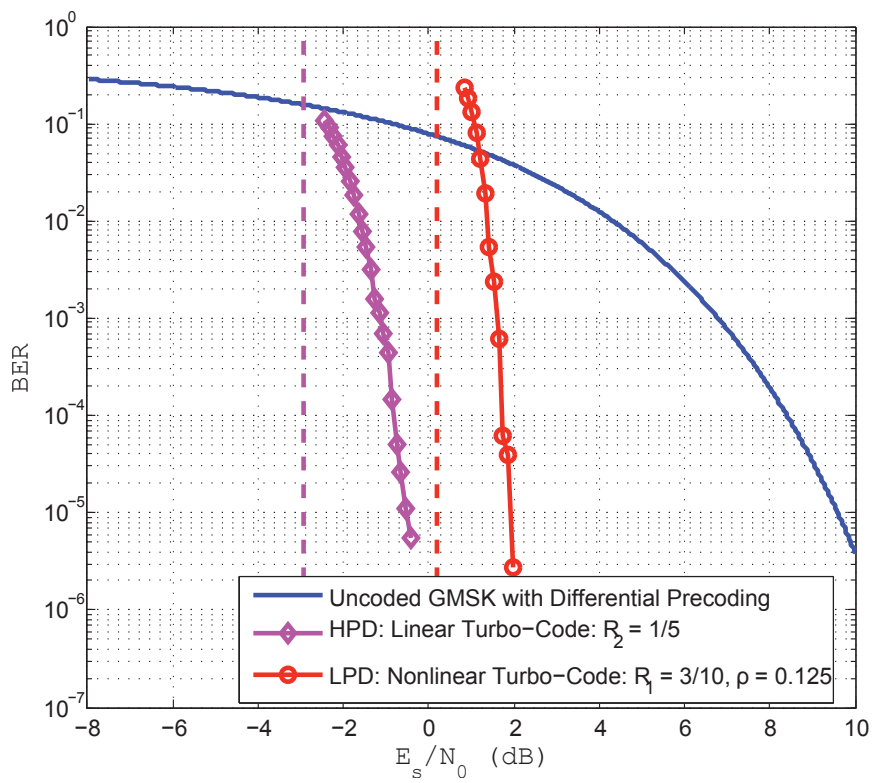

Fig. 8. BER Curves for the HPD and LPD codes at rates $R_{2}=1 / 5$ and $R_{1}=3 / 10$ respectively versus $E_{s} / N_{0}$. Theoretical capacities $\left(\eta_{1}\right.$ and $\left.\eta_{2}\right)$ for the respective codes are given by vertical dashed lines.

\section{Remarks}

Our simulations and capacity calculations assumed harddecisions from the demodulator. It is also possible to design decoders which take advantage of soft information provided by, for example, a soft-output Viterbi algorithm such as proposed in [13]. Based on our demonstrations employing hard-decisions, our results suggest that similarly optimal performance might be achieved in the soft-decoding regime.

\section{Conclusions}

For many channels, superposition coding is an optimal technique for constructing a single transmission that provides a high data rate when channel quality is good and still provides some information when the channel quality is poor (due to jamming, shadowing, etc.).

Typical superposition coding schemes for the additive white Gaussian noise channel combine signals after the modulator. For constant-envelope modulation schemes such as GMSK, which are common in tactical waveforms, this standard form of additive superposition is unacceptable because it does not preserve the original modulated constellation. This paper introduced a new approach, in which newly designed nonlinear turbo-codes are superposed prior to the modulation.

These codes were demonstrated to operate very close to the boundary of the achievable rate region for the broadcast binary symmetric channel (which results from a binary modulation scheme coupled with a hard-decision demodulator). Our results also suggest that similarly optimal performance might be obtainable in the soft decoding regime, which is one possible direction for further work.

\section{ACKNOWLEDGEMENT}

The authors would like to thank Carlos Chavez and Kent Benson (both of Rockwell Collins) for the discussions that led to this work.

\section{REFERENCES}

[1] T. M. Cover, "Broadcast channels," IEEE Trans. Inform. Theory, vol. "IT-18", pp. 2-14, January 1972.

[2] A. D. Wyner, "A theorem on the entropy of certain binary sequences and applications:Part II," IEEE Trans. Inform. Theory, vol. IT-19, pp. 772-777, Nov. 1973.

[3] H. Witsenhausen, "Entropy inequalities for discrete channels," IEEE Trans. Inform. Theory, vol. IT-20(5), pp. 610-616, September 1974.

[4] T. Kasami, S. Lin, V. K. Wei, and S. Yamamura, "Coding for the binary symmetric broadcast channel with two receivers," IEEE Trans. Inform. Theory, vol. IT-31, pp. 616-625, September 1985.

[5] U. Bhat, D. Fertonani, and T. M. Duman, "New Capacity-Achieving Encoding Schemes for Degraded Binary Broadcast Channels," in GLOBECOM '10. IEEE Global Telecomm. Conf., Miami, FL, Dec. 2010.

[6] B. Xie and R. D. Wesel, "Optimal Independent-Encoding Schemes for Several Classes of Discrete Degraded Broadcast Channels," ArXiv:0811.4162v2, 2009.

[7] — - "Optimal Natural Encoding Scheme for Discrete Multiplicative Degraded Broadcast Channels," in IEEE International Conf. on Info. Thy., Seoul, Korea, June 2009.

[8] P. P. Bergmans, "Random coding theorem for broadcast channels with degraded components," IEEE Trans. Inform. Theory, vol. 19, pp. 197207, January 1973.

[9] R. G. Gallager, "Capacity and coding for degraded broadcast channels," Probl. Pered. Inform., vol. 10, pp. 3-14, July-Sept 1974.

[10] T. M. Cover, "An achievable rate region for the broadcast channel," IEEE Trans. Inform. Theory, vol. IT-21, pp. 399-404, 1975.

[11] J. Wang, T. Courtade, T.-Y. Chen, B. Xie, and R. Wesel, "Designing Nonlinear Turbo Codes with a Target Ones Density," arXiv:1107.1563v1 [cs.IT], 2011

[12] C. Fragouli and R. D. Wesel, "Turbo encoder design for symbol interleaved parallel concatenated trellis coded modulation," IEEE Trans. Communications, vol. 49, no. 3, pp. 425-435, Mar. 2001.

[13] J. Hagenauer and P. Hoeher, "A Viterbi Algorithm with soft-decision outputs and its applications," in IEEE Global Telecommunications Conference, Dallas, TX, USA, November 1989. 